\title{
Biochemical and Genetic Characterization of PspE and GlpE, Two Single- domain Sulfurtransferases of Escherichia coli
}

\author{
Hui Cheng, Janet L. Donahue, Scott E. Battle, W. Keith Ray and Timothy J. Larson* \\ Department of Biochemistry, Virginia Polytechnic Institute and State University, Blacksburg, Virginia 24061 USA
}

\begin{abstract}
The $p s p E$ and $g l p E$ genes of Escherichia coli encode periplasmic and cytoplasmic single-domain rhodaneses, respectively, that catalyzes sulfur transfer from thiosulfate to thiophilic acceptors. Strains deficient in either or both genes were constructed. Comparison of rhodanese activity in these strains revealed that PspE provides $85 \%$ of total rhodanese activity, with GlpE contributing most of the remainder. PspE activity was four times higher during growth on glycerol versus glucose, and was not induced by conditions that induce expression of the $p s p$ regulon. The $g l p E / p s p E$ mutants displayed no apparent growth phenotypes, indicating that neither gene is required for biosynthesis of essential sulfurcontaining molecules. PspE was purified by using cation exchange chromatography. Two distinct active peaks were eluted and differed in the degree of stable covalent modification, as assessed by mass spectrometry. The peak eluting earliest contained the equivalent mass of two additional sulfur atoms, whereas the second peak contained mainly one additional sulfur. Kinetic properties of purified PspE were consistent with catalysis occurring via a double-displacement mechanism via an enzyme-sulfur intermediate involving the active site cysteine. $K_{m}$ s for $\mathrm{SSO}_{3}{ }^{2-}$ and $\mathrm{CN}^{-}$were $2.7 \mathrm{mM}$ and $32 \mathrm{mM}$, respectively, and $k_{\text {cat }}$ was $64^{\mathrm{s}-1}$. The enzyme also catalyzed transfer of sulfur from thiosulfate to dithiothreitol, ultimately releasing sulfide.
\end{abstract}

\section{INTRODUCTION}

The enzyme rhodanese catalyzes the transfer of sulfur from thiosulfate to cyanide, producing thiocyanate and sulfite (for reviews, see [1,2]. Catalysis occurs via a ping-pong mechanism, with an active site cysteine residue of the enzyme carrying the transferred sulfur as a covalent intermediate (a cysteine persulfide). In the absence of cyanide, the covalently bound sulfur may be transferred to other thiophilic acceptors, such as thiols. When a dithiol is the acceptor substrate, sulfide and the oxidized disulfide are the products of the reaction. Bovine rhodanese was discovered first, and a variety of enzymological and structural studies have been performed on that and other similar enzymes [3-5]. The enzyme is comprised of two domains ( 120 amino acids each) of similar structure that have limited amino acid sequence similarity to one another. The active site cysteine is only present within the carboxy-terminal rhodanese domain. Depending on the nature of the amino acids present in the active site loop, the tandem domain enzymes are classified as thiosulfate:cyanide sulfurtransferases, or as mercpatopyruvate sulfurtransferases [4].

Although rhodanese was discovered more than 70 years ago, physiological functions of various rhodaneses are just now being elucidated. Since the enzyme uses thiosulfate to convert toxic cyanide to the less toxic thiocyanate plus sulfite, it has been proposed that rhodaneses participate in cyanide detoxification $[2,6,7]$. It is likely, however, that proteins containing a rhodanese domain play a variety of roles in trafficking of sulfur (and selenium) during synthesis of other cellular metabolites. Genome sequence information reveals that almost all organisms encode multiple rhodaneses, some

*Address correspondence to this author at the Department of Biochemistry, Virginia Polytechnic Institute and State University, Blacksburg, Virginia 24061 USA; E-mail: tilarson@ vt.edu of which are tandem-domain rhodaneses. Other proteins consist of only a single rhodanese domain, and are fully capable of catalysis [8,9]. Finally, the rhodanese domain is predicted to be fused to a variety of other proteins. Knowledge of the functions of fusion partners, as well as of the genomic context of rhodanese-encoding genes in bacterial genomes has revealed the functions of some of the rhodanese domains. For example, the Thil protein of Escherichia coli (needed for thiamin biosynthesis and for insertion of sulfur into uridine of certain tRNAs) contains a C-terminal rhodanese fusion, which was found to be essential for incorporation of the sulfur atom into 4-thiouridine of tRNAs [10,11]. Another rhodanese fusion, human MOCS3, contains a C-terminal rhodanese domain that participates in sulfur transfer during molybdopterin biosynthesis [12,13]. Bacterial MoeB/ThiF proteins correspond to human MOCS3 and are found with or without a C-terminal rhodanese domain, depending on the organism and metabolic system. It is possible that a rhodanese is generally needed for biosynthesis of molybdopterin and certain other sulfur-containing molecules. MoeB/ThiF proteins lacking the rhodanese domain may require a standalone rhodanese protein to provide sulfur transfer function.

Knowledge of the genomic context can often provide clues regarding function. YbbB of $E$. coli contains an $\mathrm{N}$ terminal rhodanese domain and a C-terminal domain with an ATP-binding motif. In some organisms, $y b b B$ is present within the selD operon. selD encodes selenophosphate synthetase, suggesting that $y b b B$ might also be involved in selenium metabolism. It was found that the rhodanese domain of YbbB is necessary for incorporation of selenium into tRNA. YbbB catalyzes the conversion of 2-thiouridine to 2selenouridine at the wobble position of certain tRNAs [14]. There are other examples where a rhodanese gene is found within an operon or cluster of genes known to be involved in sulfur metabolism. For example, the pathways for biosyntheses of pyridine-2,6-bis(thiocarboxylate) in Pseudomonas 
stutzeri and thioquinolobactin in P. fluorescens are dependent on enzymes similar to those involved in molybdopterin biosynthesis, and the gene clusters encoding these enzymes also encode a rhodanese $[15,16]$. Therefore, it is likely that these rhodaneses participate in sulfur transfer in a manner analogous to the rhodanese domain fused to the $\mathrm{C}$-terminus of human MOCS3 [12].

In addition to thiI and $y b b B$, the genome of $E$. coli contains seven other genes that are predicted to encode proteins with one or more rhodanese domains. Four of them (yceA, $y g a P, y i b N$ and $y n j E$ ), or their corresponding proteins, have not been studied. The other three proteins (GlpE, PspE and SseA) have known enzymatic functions and have been characterized in some detail $[4,8,9,17,18]$, but their physiological functions are not known. GlpE and PspE are single-domain rhodaneses, and SseA is a tandem-domain mercaptopyruvate sulfurtransferase. GlpE is encoded by the first gene in the complex glpEGR operon [19], which also encodes a rhomboid protein (GlpG [20]) and the repressor (GlpR) for the glycerol 3-phosphate regulon [21]. This genomic context suggests that a rhodanese might participate in the regulatory functions exerted by GlpG or GlpR, but it was found that the complex glpEGR operon is not subject to regulation by the $g l p R$-encoded repressor, placing in doubt the possibility that GlpE plays a role in the utilization of glycerol 3-phosphate. PspE, a periplasmic protein, is reported to be a member of the pspABCDE (phage shock protein) operon [22,23]. Yet it is not clear what role a periplasmic sulfurtransferase might play in the cellular stress response to filamentous phage infection or other stresses, such as high salt, heat shock, or organic solvents, which are also known to induce this operon [24,25].

In the work reported here, genetic and biochemical approaches were used to show that $g l p E$ is apparently dispensable for function of the glycerol 3-phosphate regulon. In addition, neither $g l p E$ nor $p s p E$ are essential for production of sulfur-containing cofactors or amino acids. Finally, the $p s p E$ gene does not appear to be coregulated with other members of the phage shock operon, but is regulated by the nature of the carbon source present in the growth medium.

\section{MATERIALS AND METHODOLOGY}

\section{Materials}

Unless specified otherwise, the reagents used were of highest quality feasible, generally obtained from New England Biolabs, Sigma-Aldrich or Fisher Scientific.

\section{Bacterial Strains}

The bacterial strains used or constructed are described in Table 1. Strain DH5 $\alpha$ Z1 was used as a host for construction of recombinant plasmids.

\section{Media and Growth Conditions}

Cultures were grown aerobically at $37^{\circ} \mathrm{C}$ in Luria-Bertani broth (LB) [26] in the presence of antibiotic as appropriate $(100 \mu \mathrm{g} / \mathrm{ml}$ ampicillin (Ap), $25 \mu \mathrm{g} / \mathrm{ml}$ kanamycin $(\mathrm{Km})$ or 10 $\mu \mathrm{g} / \mathrm{ml}$ tetracycline (Tc)). For minimal medium, M9 salts [26] were supplemented with $5 \mu \mathrm{g}$ of thiamin per $\mathrm{ml}, 0.4 \%$ glucose or glycerol as indicated, and the appropriate antibiotics.

Table 1. E. coli Strains

\begin{tabular}{|c|c|c|}
\hline MG1655 & $\mathrm{F}^{!} i l v G r f b-50 r p h-1 \lambda !$ & [29] \\
\hline DH5 $\alpha \mathrm{Z} 1$ & 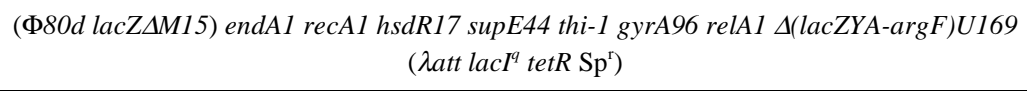 & [31] \\
\hline TL524 & MG1655 $\Delta($ lacZYA-argF $) U 169$ & {$[33]$} \\
\hline TST3 & MC4100 malT::Tn10 & {$[34]$} \\
\hline BW25113 & $\begin{array}{l}\text { lacI }^{q} \mathrm{rrnB}_{T 14} \Delta l a c Z_{W J 16} h s d R 514 \\
\quad \triangle a r a B A D_{A H 33} \Delta r h a B A D_{L D 78}\end{array}$ & {$[35]$} \\
\hline HC6.1 & TL524 $\Delta p s p E:: \mathrm{Km}^{\mathrm{r}} F R T$ & $\mathrm{P} 1(\mathrm{HC} 1.1 \rightarrow \mathrm{TL} 524)$ \\
\hline $\mathrm{HC7.1}$ & TL524 $\triangle p s p E:: F R T^{\mathrm{a}}$ & FLP removal of $\mathrm{Km}^{\mathrm{r}}$ from HC6.1 \\
\hline $\mathrm{HC} 8.1$ & TL524 $\Delta p s p E:: F R T \Delta g l p E:: \mathrm{Km}^{\mathrm{r}} F R T$ & $\mathrm{P} 1(\mathrm{AL} 1 \rightarrow \mathrm{HC} 7.1)$ \\
\hline JLD17201 & TL524 $\Delta g l p E:: \mathrm{Km}^{\mathrm{r}} F R T$ & $\mathrm{P} 1(\mathrm{AL} 1 \rightarrow \mathrm{TL} 524)$ \\
\hline JLD17204 & TL524 $\Delta g l p E:: F R T^{\text {a }}$ & FLP removal of $\mathrm{Km}^{\mathrm{r}}$ from JLD17201 \\
\hline FA035 & TL524 $\Delta p s p E:: F R T \Delta g l p E:: F R T^{\mathrm{a}}$ & FLP removal of $\mathrm{Km}^{\mathrm{r}}$ from $\mathrm{HC} 8.1$ \\
\hline
\end{tabular}

${ }^{a} A$ 113-bp FRT "scar" remains following removal of the $\mathrm{Km}^{\mathrm{r}}$ cassette by FLP recombinase [35]. 
For testing of potential auxotrophies, cultures were grown overnight in liquid minimal medium containing low levels of supplements, harvested, washed four times with medium lacking the vitamin to be tested, and then diluted 500 fold into minimal medium lacking the vitamin [27]. Growth was followed by monitoring optical density for $24 \mathrm{~h}$. To test the ability of sulfurtransferase mutants to synthesize molybdopterin, mutants were cultured anaerobically on LB containing $20 \mathrm{mM}$ sodium chlorate. Molybdopterin-deficient strains are chlorate resistant, since they are unable to assemble active nitrate reductase [28]. Nitrate reductase is capable of converting chlorate to chlorite, which is toxic. For all phenotypic testing, control strains harboring mutations in one of the known biosynthetic genes were used as controls.

\section{Construction of Plasmid pFRT-K and pSB1}

A $\mathrm{Km}^{\mathrm{r}}$ cassette, flanked by directly repeated FRT sites, was obtained from pCP15 [36] and cloned as an EcoRIHindIII fragment into the same sites of pBluescript- $\mathrm{KS}^{+}$to create pFRT-K [37]. Plasmid pPS-3, provided by Peter Model (The Rockefeller University) contains the $p s p$ operon on a $4.5 \mathrm{~kb} E c o$ RI fragment in pBluescript. The pspE gene was subcloned from pPS-3 [38] as a 723 bp SalI-EcoRI fragment into the same sites of pBluescript- $\mathrm{KS}^{+}$to create pSB1.

\section{Deletion of the Chromosomal pspE Gene}

A plasmid in which most of the $p s p E$ gene was replaced by a $\mathrm{Km}^{\mathrm{r}}$ FRT cassette was constructed by cloning DNA fragments that flank $p s p E$ into pFRT-K such that these regions flank the $\mathrm{Km}^{\mathrm{r}}$ FRT cassette. The 5' flanking (412 bp on a XhoI-DraI fragment) and 3' flanking regions (115 bp on a PshAI-BamHI fragment) of $p s p E$ were obtained from pSB1 and cloned into the XhoI-HincII and SmaI-BamHI sites of pFRT-K, respectively, yielding pHCE2 in which codons 371 of $p s p E$ gene were deleted. The method of Datsenko and Wanner [35] was used for replacing the wild-type $p s p E$ allele of strain BW25113(pKD46) with the $\Delta p s p E:: \mathrm{Km}^{\mathrm{r}} F R T$ allele of pHCE2 to create strain HC1.1. Using bacteriophage P1mediated transduction [34], $\Delta p s p E:: \mathrm{Km}^{\mathrm{r}} F R T$ was moved into strain TL524 for testing of potential phenotypes (strain HC6.1). A kanamycin-sensitive derivative of HC6.1 (named HC7.1) was obtained by Flp-mediated excision of the $\mathrm{Km}^{\mathrm{r}}$ cassette of $\Delta p s p E:: \mathrm{Km}^{\mathrm{r}} F R T$ using pCP20 [36]. The $p s p E$ deletion/replacement alleles of both strains were verified by PCR analysis using appropriate primers and chromosomal DNA of strains HC6.1 and HC7.1 as templates. In addition, the $\mathrm{Km}^{\mathrm{r}}$ cassette was mapped to the expected chromosomal location by demonstrating that it was $98 \%$ cotransducible with the zcj-233::Tn10 allele of strain CAG12028 during phage P1-mediated transduction. This Tn 10 is located within 1200 bp of $p s p E$ [32].

\section{Deletion of the Chromosomal glpE Gene}

The $g l p E$ gene was deleted in a manner analogous to that described above for $p s p E$. Plasmid pJAB1 was constructed with appropriate restriction fragments cloned on either side of the $\mathrm{Km}^{\mathrm{r}}$ FRT cassette of pFRT-K such that $204 \mathrm{bp}$ of $g l p E$ were replaced, beginning at the BstBI site within codon 5 of glpE (nucleotides 3559629-3559833 of the E. coli chromosome, GenBank LOCUS NC_000913; details available upon request). E. coli host strain BW25113(pKD46) was trans- formed with linearized pJAB1 with selection for kanamycin resistance. The position of the $\Delta g l p E:: \mathrm{Km}^{\mathrm{r}} F R T$ allele of one transformants, strain AL1, was found to be at the expected location by P1-mediated cotransduction with the malT::Tn 10 allele of strain TST3 (70\% cotransduction observed). The $\triangle g l p E:: \mathrm{Km}^{\mathrm{r}} F R T$ allele of AL1 was moved by $\mathrm{P} 1$ transduction into strains TL524 and HC7.1 by selection for kanamycin resistance, resulting in strains JLD17201 and HC8.1, respectively. The $\mathrm{Km}^{\mathrm{r}}$ cassette was excised from these strains by FLP-mediated recombination [36], resulting in strains JLD17204 and FA035 (Table 1). The $\Delta g l p E:: \mathrm{Km}^{\mathrm{r}}$ $F R T$ and $\triangle g l p E:: F R T$ alleles were verified by PCR.

\section{Construction of PspE Overexpression Plasmid pHC4.1}

The pspE gene was amplified from genomic DNA of strain MG1655 by PCR using the primers pspE1H1, ccatagaaggacgctCaTatgtttaa and pspE2H1, cgctcatggtgaattcttttaa cct (stop codon in italics), where the underlined letters indicate restriction sites for $N d e \mathrm{I}$ and EcoRI, and the uppercase letters are mismatches. After cleavage with NdeI and EcoRI, the amplified product was cloned into the same sites of pT77 [30], yielding pHC4.1.

\section{Purification of PspE}

Strain BL21(DE3)(pHC4.1) was grown in $750 \mathrm{ml}$ of LBampicillin to an $\mathrm{OD}_{600}$ of $\sim 0.5$, and expression of PspE was induced for 2 to $4 \mathrm{~h}$ by the addition of $0.5 \mathrm{mM}$ IPTG $\left(\mathrm{OD}_{600}\right.$ of $\sim 1)$. Cells were harvested $\left(4{ }^{\circ} \mathrm{C}\right)$ by centrifugation and washed with $30 \mathrm{ml}$ of $20 \mathrm{mM}$ sodium acetate, pH 5 (buffer A). The cell pellet was stored at $-70{ }^{\circ} \mathrm{C}$. Freeze-thaw extraction of PspE was based on the same method used for purification of GlpE [8]. Frozen cells were thawed on ice, suspended in $15 \mathrm{ml}$ of buffer $\mathrm{A}$, and incubated on ice for 30 min. Cell debris was removed from the extract by centrifugation at $40,000 \times \mathrm{g}$ for $90 \mathrm{~min}$. The freeze-thaw extract was loaded onto a prepacked Waters propylsulfonic acid (SP) cation-exchange column $(1 \times 10 \mathrm{~cm})$ equilibrated at room temperature with buffer A. After extensive washing with buffer A, the column was developed with a 55-ml linear gradient of 0 to $825 \mathrm{mM} \mathrm{NaCl}$. Two peaks containing rhodanese activity (PspE1 and PspE2), eluting between 330 and 600 $\mathrm{mM} \mathrm{NaCl}$, were pooled separately, adjusted to $\mathrm{pH} 7.0$ by addition of $20 \mu \mathrm{l}$ of $1 \mathrm{M}$ Tris- $\mathrm{HCl}(\mathrm{pH} 8.0)$ per $\mathrm{ml}$ of collected fraction, and stored in aliquots at $4{ }^{\circ} \mathrm{C}$.

\section{Polyacrylamide Gel Electrophoresis}

Sodium dodecyl sulfate-polyacrylamide gel electrophoresis was performed as described by Laemmli [39] on $15 \%$ polyacrylamide gels.

Non-denaturing polyacrylamide gel electrophoresis was performed using the same gel and buffer system as above, but lacking SDS. The sample loading buffer contained 62.5 $\mathrm{mM}$ Tris- $\mathrm{HCl}$ (pH 6.8), 10\% glycerol, $0.03 \%$ bromophenol blue. Gels were pre-electrophoresed for $1 \mathrm{~h}$.

\section{Assay of Sulfurtransferase Activities}

The standard rhodanese assay was essentially that described previously to quantify thiocyanate $[8,40]$. Assays $(0.5 \mathrm{ml})$ contained $100 \mathrm{mM}$ Tris-acetate $(\mathrm{pH} 8.6), 10 \mathrm{mM}$ ammonium thiosulfate, $50 \mathrm{mM} \mathrm{KCN}$, and enzyme. Reactions were initiated by the addition of $\mathrm{KCN}$ and terminated, after 2 
min, by the addition of $0.25 \mathrm{ml}$ of $15 \%$ formaldehyde, followed by $0.75 \mathrm{ml}$ of ferric nitrate reagent. The absorbance at $460 \mathrm{~nm}$ was determined and compared to a standard curve for thiocyanate. One unit of activity is defined as the amount of enzyme that catalyzes the production of $1 \mu \mathrm{mol}$ of thiocyanate per min at $25{ }^{\circ} \mathrm{C}$. For kinetic studies, the concentration of the Tris-acetate buffer was increased to $200 \mathrm{mM}$, which helped maintained constant $\mathrm{pH}$ as substrate concentrations were varied.

Standard assays (1-ml) employing dithiothreitol as sulfur acceptor contained the same components as above except that $10 \mathrm{mM}$ dithiothreitol replaced $\mathrm{KCN}$. Absorbance at 283 $\mathrm{nm}$ was monitored continuously and activity was calculated based on an extinction coefficient of $273 \mathrm{M}^{-1} \mathrm{~cm}^{-1}$ for oxidized dithiothreitol.

Mercaptopyruvate sulfurtransferase activity catalyzed by PspE was assayed using two methods. The first was a modification of the assay used for rhodanese with substitution of $50 \mathrm{mM}$ mercaptopyruvate for thiosulfate. Reactions were initiated by the addition of enzyme and terminated after a 15min incubation at $25^{\circ} \mathrm{C}$. The second assay used $10 \mathrm{mM} \mathrm{3-}$ mercaptopyruvate as the sulfur donor and $25 \mathrm{mM} \mathrm{2-}$ mercaptoethanol as sulfur acceptor. The production of pyruvate was measured following its reaction with 2,4dinitrophenylhydrazine [41].

\section{Determination of Cyanolyzable Sulfur of PspE}

Persulfide sulfur bound to cysteine residues of proteins can be quantitatively released by treatment with $\mathrm{CN}^{-}$and quantified as $\mathrm{SCN}^{-}$[42]. The two peaks of PspE obtained from cation-exchange chromatography were adjusted to $\mathrm{pH}$ 7.0 by addition of $1 \mathrm{M}$ Tris- $\mathrm{HCl} \mathrm{pH} 8.0$, because persulfide sulfur is acid labile [43]. Various amounts of PspE were treated with $100 \mathrm{mM}$ cyanide for $5 \mathrm{~min}$ at room temperature. Thiocyanate was then quantified as the ferric thiocyanate complex as described for the rhodanese assay.

\section{Analysis of Covalent Modification of PspE by Mass Spec- trometry}

Aliquots of PspE1 and PspE2 $(20-50 \mathrm{nmol}$ in $0.5 \mathrm{ml})$ eluted from the cation exchange column were thoroughly dialyzed against $10 \mathrm{mM}$ Tris- $\mathrm{HCl}(\mathrm{pH} \mathrm{7.5)}$. An aliquot of PspE2 was treated with $1 \mathrm{mM}$ ammonium thiosulfate for 15 $\mathrm{min}$ at room temperature. The samples were prepared for mass spectrometry by precipitation with four volumes of icecold methanol $\left(-70{ }^{\circ} \mathrm{C}\right.$, overnight). Precipitated proteins were washed with cold $70 \%$ methanol, dried, and stored at 4 ${ }^{\circ} \mathrm{C}$. Protein pellets were suspended in $50 \%$ methanol, $10 \%$ formic acid at approximately $20 \mathrm{pmol} / \mu \mathrm{L}$. Samples were analyzed using a Thermo Electron TSQ Quantum AM mass spectrometer equipped with an electrospray source. Injection was at 3-5 $\mu \mathrm{L}$ per min using a $250 \mu \mathrm{L}$ Hamilton gas-tight syringe. Spray voltage was 4000 volts, sheath gas pressure was 10 psi, capillary tube temperature was $270{ }^{\circ} \mathrm{C}$ (tube lens offset was 160 and the lens offset was -1.3 . The machine had been tuned and calibrated using caffeine as a standard). The $\mathrm{m} / \mathrm{z}$ range from 550 to 1500 was scanned using Q1 with a 1.5 second scan time and a peak width of 0.7 . Data were collected for approximately 5 to $10 \mathrm{~min}$ and the spectra were averaged using Xcalibur software. Deconvolution of the averaged spectra was performed manually.

\section{RESULTS}

\section{Isolation and Characterization of $p s p E$ and $g l p E$ Mutants}

In order to assess the relative contributions of the two single-domain rodaneses to cellular sulfurtransferase activity, and possibly to cellular physiology, strains deficient in either $p s p E, g l p E$ or both were constructed. In each case, the majority of the gene was ultimately replaced by the FRT scar. All insertion and deletion alleles were verified by PCR, and were mapped to the expected chromosomal locations by using phage P1-mediated cotransduction with an independent neighboring allele.

Growth phenotypes of the three mutant strains were compared with the wild type using rich and minimal media. All four strains were capable of growth on glucose, glycerol or acetate minimal media without supplementation. Growth on minimal medium indicated that neither $g l p E$ nor $p s p E$ is required for production of sulfur containing amino acids (cysteine and methionine) or cofactors (thiamin, biotin, lipoate). To find out if either gene is required for molybdopterin biosynthesis, the strains were plated on rich medium containing chlorate and incubated under anaerobic conditions. Under these conditions, strains unable to produce molybdopterin display a chlorate-resistant phenotype, since they are unable to produce active nitrate reductase (nitrate reductase converts chlorate to chlorite, which is toxic [28]). All four strains displayed equal sensitivity toward chlorate, indicating that $p s p E$ and $g l p E$ are not essential for production of molybdopterin.

The periplasmic location of PspE suggested that it might serve as a detoxification enzyme for cyanide or for heavy metal cations such as cadmium. However, the $p s p E$ mutant HC7.1 was not more sensitive to $\mathrm{KCN}(0-1 \mathrm{mM})$ when compared to the wild type during growth on LB. Addition of 1 $\mathrm{mM}$ thiosulfate had no influence on the shapes of the growth curves. The results suggest that PspE does not play a role in cyanide detoxification, and are in agreement with those of Adams et al. [9], who reported that overexpression of PspE did not confer resistance to cyanide.

The periplasmic Dsb (disulfide bond formation) proteins, DsbA and DsbB, are involved in $\mathrm{Hg}^{2+}$ and $\mathrm{Cd}^{2+}$ detoxification by an unknown mechanism $[44,45]$. Although the Dsb proteins generally function to maintain disulfide bonds in periplasmic proteins, the possibility that the flow of electron could be from periplasmic dithiol protein, through the Dsb system to the persulfide form of periplasmic PspE was considered. Reduction of the cysteine persulfide would result in formation of hydrogen sulfide, which would precipitate (thereby detoxifying) heavy metal cations at the cell surface. If this were the case, mutation of $p s p E$ would be predicted to increase the sensitivity of $E$. coli to $\mathrm{Hg}^{2+}$ and $\mathrm{Cd}^{2+}$. Growth of wild-type and mutant strains deficient in $p s p E$ was compared on solid LB agar containing various concentrations of $\mathrm{CdSO}_{4}(0.01,0.03,0.1,0.3$ and $1.0 \mathrm{mM})$. Except for medium containing $1 \mathrm{mM} \mathrm{Cd}^{2+}$, all strains grew in the presence of $\mathrm{Cd}^{2+}$. No significant difference between the sulfurtransferase-deficient strains and the wild-type strain was observed.

A variety of other phenotypic tests were performed in which growth of the rhodanese-deficient strains was compared to that of the wild type. These included sensitivity to 
oxidative stress (peroxide, methyl viologen, selenite), antibiotics (tetracycline, chloramphenicol, spectinomycin, ampicillin, streptomycin, kanamycin, nalidixic acid), and detergent (deoxycholate). The ability to utilize a variety of compounds (sulfate, thiosulfate, taurine, cysteine) as sole sulfur source at relatively low concentration $(0.05-0.1 \mathrm{mM})$ was also investigated. In all cases, growth of the mutant strains was comparable to that of the wild-type strain.

\section{Rhodanese Activities in Wild-Type and pspE/glpE Mu- tant Strains}

In order to determine the contributions of PspE and GlpE to total rhodanese activity in $E$. coli, activities in cell-free extracts of the wild-type and the single and double mutant strains were determined following growth in glucose minimal medium. The results indicate that the $p s p E$ gene is responsible for contributing most of the rhodanese activity (Table 2).

Table 2. Comparison of Rhodanese Activities in Wild-Type and Sulfurtransferase-Deficient Strains

\begin{tabular}{|c|c|c|}
\hline Strain & Genotype & Sp. act. (U/mg) \\
\hline \hline TL524 & Wild type & 0.065 \\
\hline JLD17204 & $\Delta g l p E:: F R T$ & 0.066 \\
\hline HC7.1 & $\Delta p s p E:: F R T$ & 0.013 \\
\hline HC8.1 & $\begin{array}{c}\Delta p s p E:: F R T \\
\Delta g l p E:: \mathrm{Km}^{\mathrm{r}} F R T\end{array}$ & 0.003 \\
\hline
\end{tabular}

${ }^{a}$ Cultures were grown in minimal glucose medium at $37^{\circ} \mathrm{C}$ and harvested at an $\mathrm{OD}_{600}$ of 1.0. Specific activities were determined using crude extracts derived from sonicated cells and are expressed as units per mg protein. Substrate concentrations employed were $50 \mathrm{mM}$ thiosulfate and $50 \mathrm{mM}$ cyanide.

In various experiments, it was found that deletion of $p s p E$ decreased total rhodanese activity by $80-90 \%$, whereas deletion of $g l p E$ resulted in a decrease of about 10 $\%$. Only $5 \%$ of total rhodanese activity remained upon deletion of both genes. Residual activity could be due to the presence of one or more of the other proteins harboring a rhodanese domain.

\section{Regulation of $p s p E$ and $g l p E$ Expression}

Clues regarding function of the sulfurtransferases of $E$. coli were sought by investigating global gene expression data available in ASAP [46]. These data indicated that $p s p E$ mRNA levels were expressed at higher levels during growth on glycerol, acetate or proline as carbon source relative to expression during growth on glucose. The expression of $g l p E$ was about the same regardless of carbon source. To determine if the apparent increased content of $p s p E$ mRNA during growth on glycerol minimal medium is reflected in an increase in rhodanese activity, an overnight culture of strain JLD17204 $(\Delta g l p E)$ was diluted into fresh minimal medium containing either glycerol or glucose as carbon source. At timed intervals, aliquots were withdrawn for measurement of optical density and rhodanese activity. Rhodanese activity was induced four to five fold during exponential growth on glycerol relative to the level of activity found in cells growing on glucose (Fig. 1).

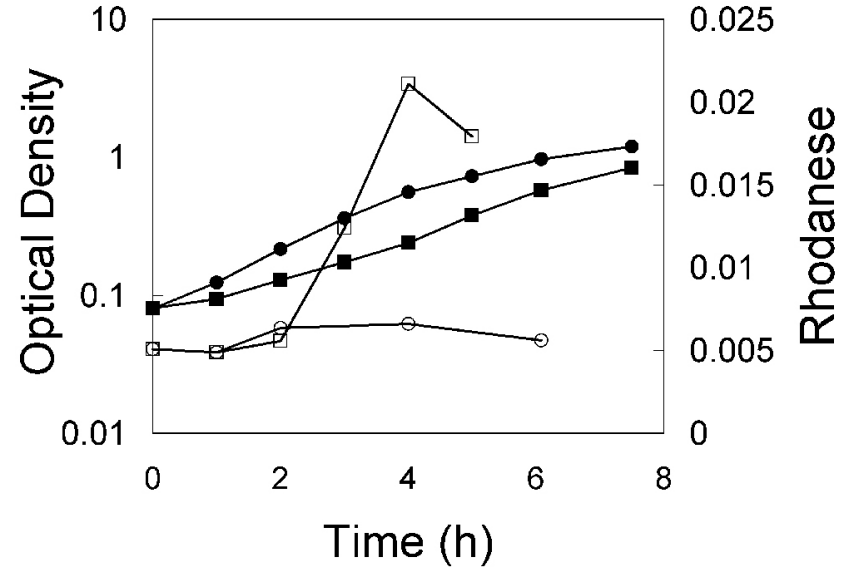

Fig. (1). Rhodanese activities during growth on glycerol or glucose minimal medium. Strain JLD17204 ( $\triangle g l p E:: F R T)$ was grown overnight in minimal medium with glucose. Cells were harvested by centrifugation, washed, and then diluted into medium with either glucose (circles) or glycerol (squares). The optical density at 600 $\mathrm{nm}$ (closed symbols) and rhodanese activity (open symbols) were monitored. Rhodanese activity was determined using intact cells harvested at the indicated time points and corresponds to the activity (units) found in the equivalent of $1 \mathrm{ml}$ of cells at an OD of 2 . Substrate concentrations of $50 \mathrm{mM}$ thiosulfate and $50 \mathrm{mM}$ cyanide were employed for these assays.

Induction of rhodanese activity was due to an increase in $p s p E$ expression, since the phenomenon did not occur in the strains in which $p s p E$ was deleted (Fig. 2).

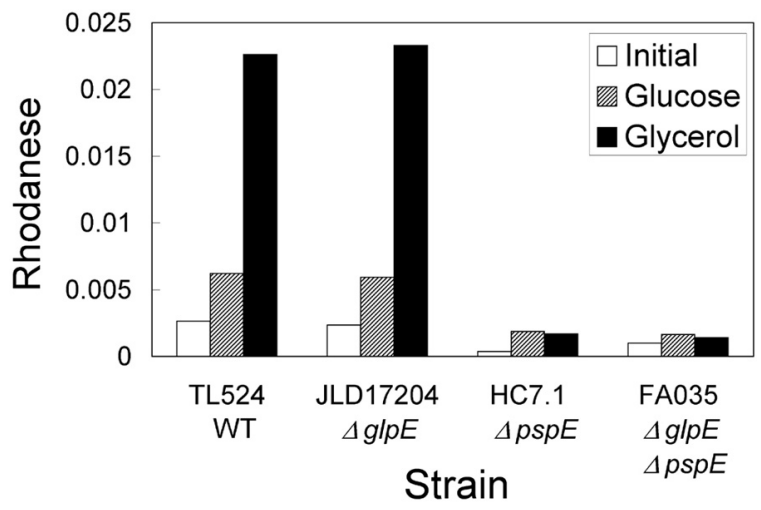

Fig. (2). Rhodanese activity in wild-type and mutant strains. The indicated strains were grown overnight in glucose minimal medium, washed and then diluted into medium containing glucose (striped bars) or glycerol (black bars). Cells were harvested during exponential growth (OD600 of 0.64-0.79) and rhodanese activities were determined as described in the legend to Fig. (1). Activities found in the overnight cultures are indicated by the open bars.

The $p s p E$ gene is part of the pspABCDE operon, but apparently is also transcribed independently from the other genes of the operon [23]. The rhodanese activity of PspE provides a facile reporter for expression of the operon. To determine if $p s p E$ is induced in response to the stressors that induce other members of the $p s p$ operon, rhodanese specific activity was determined in cultures of strain JLD17204 $(\triangle g l p E)$ growing in glucose minimal medium following various treatments (salt stress using $0.75 \mathrm{M} \mathrm{NaCl}$ or heat 
stress at $50{ }^{\circ} \mathrm{C}$ for $10 \mathrm{~min}$ ). No increase in rhodanese activity was apparent in aliquots taken at 20, 40,60 and $135 \mathrm{~min}$ following addition of $\mathrm{NaCl}$ (relative to unstressed cells). Small $(10 \%$ and $40 \%)$ increases in rhodanese activity were observed 20 and 40 min following heat stress, but then activity declined. These observations indicate that $p s p E$ is not significantly co-regulated with the other members of the pspABCD operon, whose expression increases much more dramatically following stress. Data available in ASAP suggest that pspABCD operon mRNA levels increase from 5 to 10 fold following heat stress, while $p s p E$ mRNA levels increase only 1.5 fold [47].

\section{Purification and Characterization of PspE}

PspE was previously purified as a cytoplasmically expressed protein possessing an N-terminal His-6 affinity tag [9]. Native PspE is synthesized as a precursor with a 19 amino acid $\mathrm{N}$-terminal signal sequence and is secreted to the periplasm [38,48]. Mature PspE, comprised of only 85 amino acids, is the smallest known active rhodanese. In the present work, native PspE was overexpressed and purified as described in the "Methods." Strong overexpression and its periplasmic location facilitated purification of PspE by freeze-thaw treatment, followed by cation exchange chromatography. A four-fold purification yielded approximately 5 $\mathrm{mg}$ of PspE from a 750-ml culture. Using Coomassie blue staining following SDS-PAGE, PspE was estimated to be $>95 \%$ pure (Fig. 3A), and had a specific activity of $130-$ $150 \mathrm{U}$ per $\mathrm{mg}$ of protein.
A

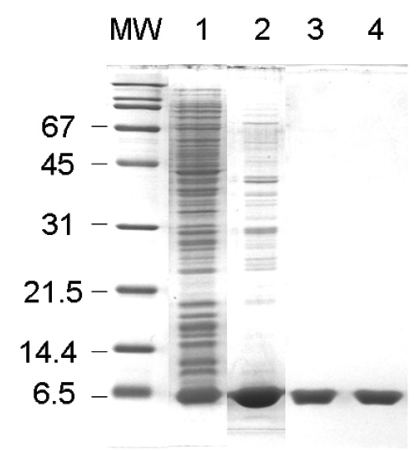

B

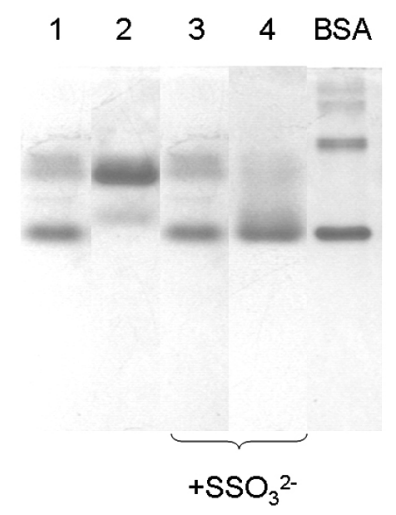

Fig. (3). Electrophoretic analysis of PspE1 and PspE2. A: Fractions obtained during purification of $\mathrm{PspE}$ from induced strain BL21(DE3)(pHC4.1) were analyzed on an SDS-polyacrylamide gel. Lane 1 , total cellular proteins $(10 \mu \mathrm{g})$. Lane 2 , freeze-thaw extract $(10 \mu \mathrm{g})$. Lanes 3 and 4 , pooled peak 1 (PspE1) and peak 2 (PspE2), respectively, from cation-exchange chromatography (3 $\mu \mathrm{g}$ each lane). MW, molecular weight standards. B. PspE1 (lanes 1 and 3) and PspE2 (lanes 2 and 4) were analyzed using nondenaturing polyacrylamide gel electrophoresis. In each lane, $3 \mu \mathrm{g}$ of PspE was analyzed. Where indicated, PspE was pre-incubated with $1 \mathrm{mM}$ ammonium thiosulfate for $5 \mathrm{~min}$ at $22{ }^{\circ} \mathrm{C}$. BSA, $2 \mu \mathrm{g}$ BSA (monomeric and dimeric forms are the two major bands) was used as a standard.

Elution of PspE from the cation exchange column using a salt gradient yielded two narrowly-separated but distinct peaks. The two peaks (PspE1 and PspE2) were collected separately and characterized. PspE1 had a more compact and/or more negatively charged form as analyzed by nondenaturing PAGE (Fig. 3B) and by gel chromatography (not shown). Treatment of PspE2 with $1 \mathrm{mM}$ ammonium thiosulfate resulted in its conversion to the faster migrating form observed in fraction PspE1 (Fig. 3B), suggesting that transfer of sulfur from thiosulfate to the active site cysteine of PspE2 results in its conversion to PspE1.

There is only one cysteine residue in PspE. Evidence for covalent modification of the active site cysteine of PspE was obtained by using several methods. First, it was found that PspE1 contained approximately one equivalent of cyanolyzable sulfur, with much less sulfur present in PspE2 (Fig. 4). Second, PspE1 was much more tolerant to chemical inactivation by the cysteine-modifying reagent 5,5'-dithio-bis(2nitrobenzoic acid) (DTNB) compared with PspE2 (data not shown). It is known that the sulfur-free form of bovine rhodanese is twice as reactive toward DTNB relative to the cysteine persulfide form of the enzyme [49], and the same appears to be the case for PspE.

Finally, analysis of the two fractions by electrospray mass spectrometry revealed that multiple, covalently modified forms of the enzyme are present in the preparations. Based upon the properties of other rhodaneses, the data are most consistent with modifications occurring on the sulfhydryl group of the active site cysteine of PspE. PspE1 was more highly modified than PspE2 (Table 3). In fact, the predominant species of PspE present in PspE1 contained the equivalent mass of either one or two sulfur atoms (along with minor amounts of more highly modified PspE). The predominant enzyme forms present in PspE2 were PspE with one sulfur atom, along with smaller amounts of unmodified PspE. A substantial fraction of both PspE1 and PspE2 apparently is present as the sulfonate-modified form (E-SSO$\left.{ }_{3} \mathrm{H}\right)$. Treatment of PspE2 with thiosulfate resulted in greater abundances of the modified forms of PspE. The observation that PspE2 released less than one equivalent of sulfur upon treatment with cyanide even though the predominant form present was the cysteine persulfide suggests that the sulfurmodified form of the enzyme is quite stable to this treatment.

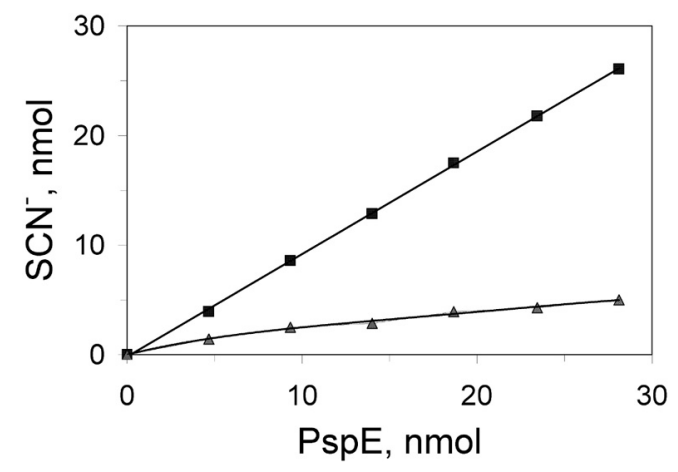

Fig. (4). Cyanolyzable sulfur of PspE1 and PspE2. Immediately following elution from the cation exchange column, fractions containing PspE1 ( $\boldsymbol{\square})$ and PspE2 ( $\boldsymbol{\Delta}$ ) were adjusted to $\mathrm{pH} 7.0$ by addition of $1 \mathrm{M}$ Tris $-\mathrm{HCl}(\mathrm{pH} \mathrm{8.0)}$ ). Aliquots were then treated with 100 $\mathrm{mM}$ cyanide for $5 \mathrm{~min}$ at room temperature. Thiocyanate was quantified as the ferric thiocyanate complex as described for the rhodanese assay. The amount of PspE was estimated using the Bradford protein assay [50], with bovine serum albumin as standard. 
Table 3. Relative Abundances and Masses of PspE Species Found in Purified Fractions PspE1 and PspE2

\begin{tabular}{|c|c|c|c|c|c|c|}
\hline \multirow{2}{*}{$\begin{array}{l}\text { Enzyme form }{ }^{\text {a }} \\
\text { (theoretical mass) }\end{array}$} & \multicolumn{6}{|c|}{ Enzyme Fraction } \\
\hline & Abundance $^{c}$ & Mass & Abundance & Mass & Abundance & Mass \\
\hline E-SH (9427.7) & 0.23 & 9427.9 & 0.60 & 9427.8 & 0.25 & 9427.6 \\
\hline E-SSH (9459.76) & 0.57 & 9459.9 & 1.00 & 9460.1 & 0.65 & 9460.3 \\
\hline E-SSO $3 \mathrm{H}(9507.76)$ & 0.67 & 9507.9 & 0.60 & 9507.8 & 1.00 & 9507.9 \\
\hline $\mathrm{E}-S S S O_{3} \mathrm{H}(9539.83)$ & $<0.1$ & -- & $<0.1$ & -- & 0.38 & 9540.5 \\
\hline
\end{tabular}

${ }^{a}$ The species of PspE observed in the mass spectral analysis are consistent with those indicated, with E-SH being the unmodified form of PspE. E-SSH is PspE containing the cys-

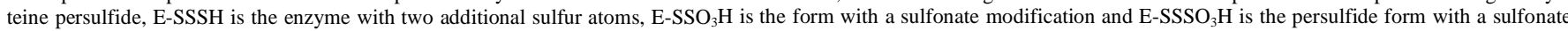
modification. The theoretical masses shown in parentheses are those calculated based on the amino acid sequence of processed (secreted) PspE as deduced from the DNA sequence.

"An aliquot of fraction PspE2 was treated with $1 \mathrm{mM}$ ammonium thiosulfate as described in "Methods."

${ }^{\mathrm{c}}$ The average abundances of the major peaks observed in the mass spectra, relative to the most abundant peak observed (designated 1.00), are shown. The molecular masses for each peak were calculated by averaging the masses obtained by deconvolution of the spectra observed for the $+10,+11,+12$ and +13 molecular ions.

Analysis of PspE by mass spectrometry confirmed that it was processed properly during its secretion to the periplasm, even when expressed at high levels. The molecular masses observed matched exactly those expected if the 19 residue signal sequence was removed.

The question arises as to the source of the covalent modifications of PspE. Thiosulfate is sometimes added to the buffers during purification of rhodaneses [8,51]. This practice insures that the active site cysteine is modified, that is, converted to its persulfide form, and as such, is more resistant to oxidation. However, we did not include thiosulfate in the buffers used during purification, and so it is likely that PspE is covalently modified in vivo, and that such modifications are relatively stable. The modifications of PspE could arise from an endogenous metabolic pathway that yields the sulfur donor, or it is possible that the growth medium contains thiosulfate or other potential sulfur donors. To determine if growth medium contains a potential sulfur source, cyanolyzable sulfur was determined in LB medium. It was found that LB contains about $0.013 \mathrm{mM}$ cyanolyzable sulfur by using PspE and a rhodanese assay run to completion. This sulfur could be the source of sulfur for modification of PspE prior to its purification by the standard protocol described above. The other possibility, that an endogenous pathway is responsible for production of a sulfur donor that modifies PspE, was tested by production of PspE during growth on a defined minimal medium that contains no added thiosulfate, but instead, only sulfate as sulfur source. PspE expression was induced during growth on M9 glucose minimal medium using strain BL21(DE3)(pHC4.1). It was found that the spent growth medium contained about $0.037 \mathrm{mM}$ sulfur donor, as assessed by the PspE-catalyzed rhodanese reaction. Furthermore, PspE produced during growth on M9-glucose eluted as two peaks during cation exchange chromatography, the first of which contained cyanolyzable sulfur (data not shown). These results show that during normal growth, $E$. coli produces compounds that provide sulfur for covalent modification of PspE with sulfane sulfur.

\section{Catalytic Properties of Purified PspE}

Purified preparations of PspE exhibited specific activities of $150-200 \mathrm{U} / \mathrm{mg}$ when assaying transfer of sulfur from thiosulfate to cyanide. Rhodaneses utilize a doubledisplacement (ping-pong) mechanism [8,52], which is also
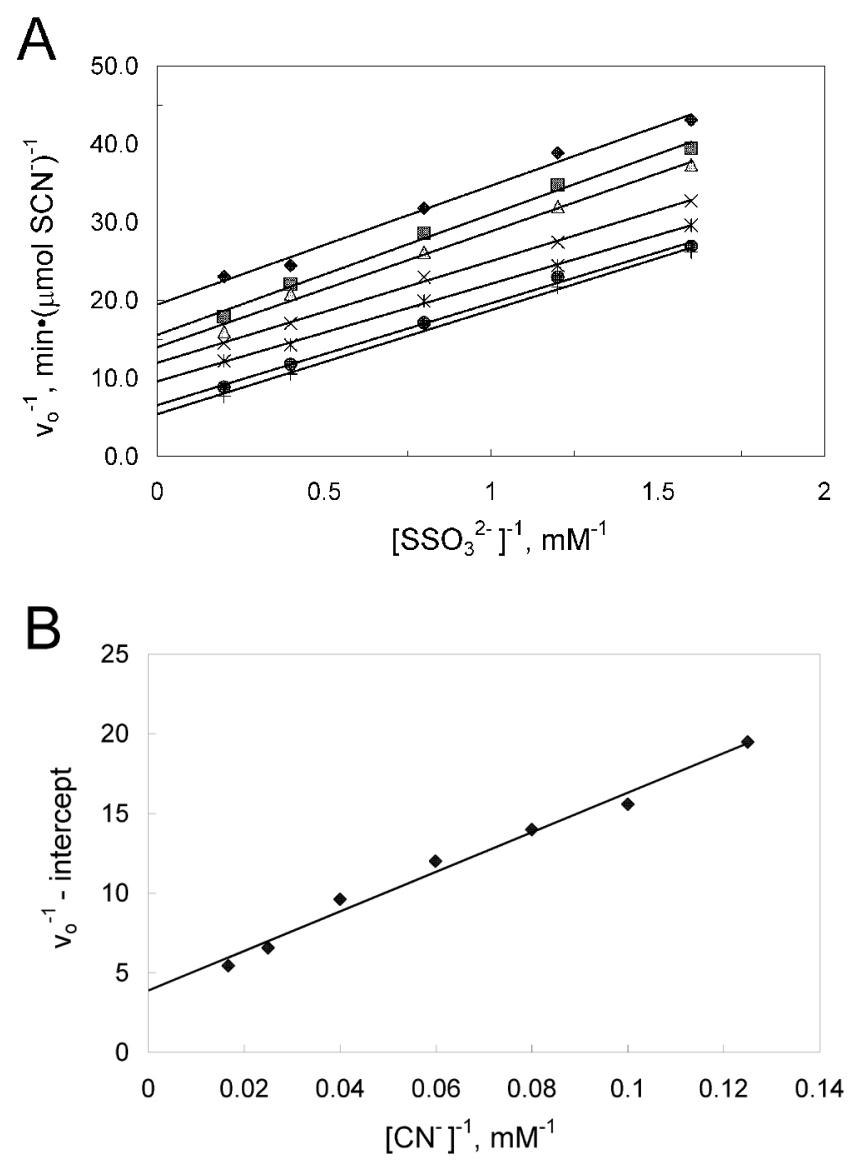

Fig. (5). Kinetic characterization of the thiosulfate:cyanide sulfurtransferase reaction catalyzed by $\mathrm{PspE}$. Each assay contained $0.63 \mu \mathrm{g}$ of purified PspE2 and $1 \mathrm{mM}$ cysteine. (A) Doublereciprocal plot of the rate of thiocyanate formation versus thiosulfate concentration at various fixed concentrations of cyanide: 8 $\mathrm{mM}(\bullet), 10 \mathrm{mM}(\boldsymbol{\square}), 12.5 \mathrm{mM}(\boldsymbol{\Delta}), 16.7 \mathrm{mM}(\times), 25 \mathrm{mM}(*), 40$ $\mathrm{mM}(\bullet), 60 \mathrm{mM}(+)$. (B) Secondary double-reciprocal plot of apparent $V_{\max }$ values from the data in panel A versus cyanide concentrations. 
apparently the case for PspE [9]. In the previous study, PspE contained an $\mathrm{N}$-terminal $\mathrm{His}_{6}$ tag, which conceivably could affect the catalytic properties of PspE. Thus, kinetic properties of PspE were reinvestigated and alternate substrates were tested as sulfur donors/acceptors. PspE2 was used to study the kinetic properties in reactions containing $1 \mathrm{mM}$ cysteine, which activates PspE activity. Data from activity measurements of purified PspE2 with various concentrations of thiosulfate at fixed concentrations of cyanide fit the equation describing a double-displacement mechanism. A plot of the data (Fig. 5) yielded $K_{m}$ values for thiosulfate and cyanide of 2.7 and $32 \mathrm{mM}$, respectively. The $V_{\max }$ value obtained was $410 \mu \mathrm{mol} \mathrm{SCN} \cdot \mathrm{min}^{-1} \cdot \mathrm{mg}^{-1}$, yielding a $k_{\text {cat }}$ of $64 \mathrm{~s}^{-}$ ${ }^{1}$. The kinetic constants were about the same as those reported previously [9], where $K_{m}$ values for thiosulfate and cyanide of 4.6 and $27 \mathrm{mM}$, respectively, were found. The small differences observed may be due to the presence or absence of the His- 6 tag, or due to differences in concentrations of thiosulfate employed in the two studies. Thiosulfate concentrations in the present study were above and below $K_{m}$ $(0.5-5 \mathrm{mM})$, whereas Adams et al. used high concentrations of thiosulfate $(10-50 \mathrm{mM})$.

As is the case for other rhodaneses, PspE also was capable of using a dithiol (dithiothreitol) as sulfur acceptor, ultimately releasing sulfide and oxidized dithiothreitol. For this reaction, the specific activity was about $300 \mathrm{U}$ per $\mathrm{mg}$ of PspE, and the apparent $K_{m}$ for dithiothreitol was about 10 $\mathrm{mM}$ when using either $4 \mathrm{mM}$ or $10 \mathrm{mM}$ thiosulfate. Finally, purified PspE possessed very low mercaptopyruvate sulfurtransferase activity. With cyanide as the sulfur acceptor (thiocyanate detection), the ratio of rhodanese to MST activity $(5 \mathrm{U} / \mathrm{mg})$ was 40 ; with 2 -mercaptoethanol as sulfur acceptor (pyruvate detection), the ratio of rhodanese to MST activity $(0.2 \mathrm{U} / \mathrm{mg})$ was 900 .

The E. coli rhodanese GlpE is inhibited only weakly by certain anions [8]. Greater inhibition by anions was observed for PspE. Addition of sodium sulfate or sodium chloride at $0.25 \mathrm{M}$ resulted in about $50 \%$ inhibition of rhodanese activity. Addition of sodium phosphate at the same concentration resulted in about $65 \%$ inhibition. Sulfite, one of the products of the rhodanese reaction, strongly inhibited PspE activity (1 $\mathrm{mM}$ sodium sulfite resulted in more than $50 \%$ inhibition of rhodanese activity, data not shown).

\section{DISCUSSION}

Characterization of strains deficient in $p s p E$ and/or $g l p E$ established that the encoded single-domain rhodaneses are the major contributors of rhodanese activity in E. coli. Strains lacking both $p s p E$ and $g l p E$ had less than $5 \%$ of the activity found in wild-type strains. Lack of rhodanese activity had no apparent influence on the growth of $E$. coli under the various conditions tested. It was hypothesized that one of the single-domain rhodaneses might participate in trafficking of sulfur during biosynthesis of sulfur-containing cofactors. For example, the MoeB protein of E. coli lacks a rhodanese domain, and so might require a stand-alone rhodanese for sulfur trafficking during molybdopterin biosynthesis, as is the case for the human enzyme [12]. However, it is apparent that neither GlpE nor PspE is essential for molybdopterin synthesis, or for synthesis of other essential sulfur-containing cofactors. It is possible that one of the other sulfurtrans- ferases present in E. coli cooperates with MoeB during molybdopterin synthesis. However, characterization of mutants deficient in each of the other rhodanese genes has not yielded further information regarding physiological function (unpublished data).

E. coli and most other organisms possess multiple proteins with the rhodanese domain. Although the nine paralogs found in $E$. coli are easily identified based on their conserved sequence motifs, they are not very similar in sequence to one another, and some of their rhodanese domains are found fused to other unique protein domains (Fig. 6). In addition, the proteins are found not only in the cytoplasm, but also in other subcellular locations (e.g., periplasm for PspE, cytoplasmic membrane for $\mathrm{YgaP}$ ). Therefore, the different paralogs are likely to have defined, unique physiological roles. As pointed out in the Introduction, two of these proteins participate in modifying tRNAs (ThiI and YbbB), but the functions of the others must still be defined.

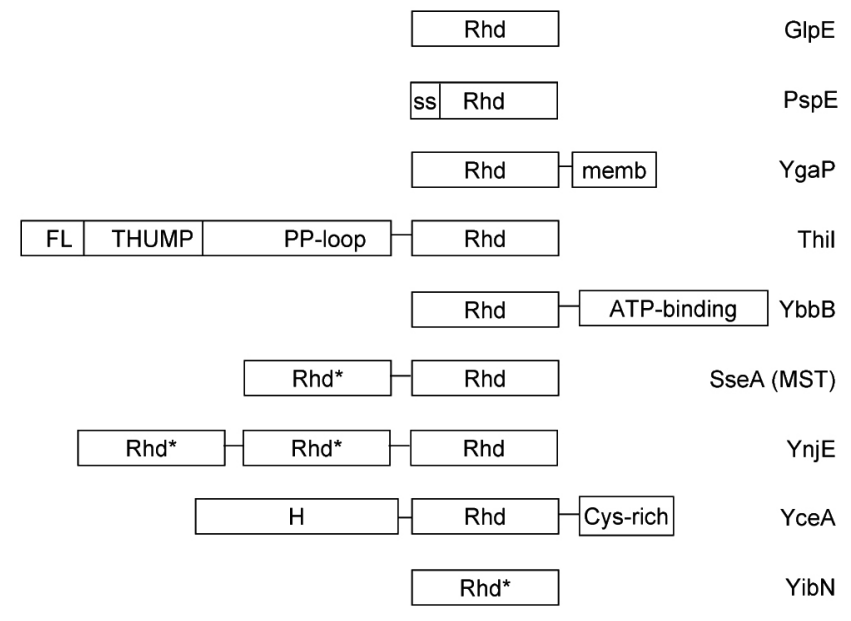

Fig. (6). Schematic illustrating rhodanese paralogs found in E. coli. The Abbreviations used are: Rhd, rhodanese homology domain; Rhd*, rhodanese homology domain in which an aspartate replaces the conserved active site cysteine of Rhd; ss, cleaved signal sequence of PspE; memb; hydrophobic segment of YgaP; FL, THUMP and PP-loop are the ferredoxin-like domain, RNA-binding domain found in RNA base-modifying enzymes, and ATP pyrophosphatase domains, respectively, of ThiI [54]; ATP-binding, domain of tRNA 2-selenouridine synthase involved in nucleotide binding, tRNA interaction and modification; $\mathrm{H}$, hypothetical domain of unknown function; Cys-rich, cysteine-rich domain of YceA.

Results presented in this paper indicate that PspE is not likely to be an essential part of the phage shock regulon. Expression of rhodanese activity was not induced by stressors that result in induction of the other members of the psp operon (heat stress, osmotic stress), but instead was induced during growth on less preferred carbon sources such as glycerol. There are no obvious simple transcription terminators preceding $p s p E$, and so it is likely that $p s p E$ expression also could be induced by signals that induce the $p s p A B C D$ operon to its highest levels (filamentous phage infection, or in regulatory mutants). Microarray analysis in which mRNA levels in a $p s p F$ (positive regulator of $p s p$ gene expression) mutant were compared with those in a $p s p A$ (negative regulator) 
Table 4. Comparison of PspE and GlpE Rhodaneses

\begin{tabular}{|c|c|c|c|c|}
\hline Enzyme & $\boldsymbol{M}_{\boldsymbol{r}}(\mathbf{A A})$ & $\boldsymbol{k}_{\text {cat }}\left(\mathbf{s}^{-\mathbf{1}}\right)$ & $\boldsymbol{K}_{\boldsymbol{m}}, \mathbf{S S O}_{\mathbf{3}}{ }^{2-}(\mathbf{m M})$ & $\boldsymbol{K}_{\boldsymbol{m}}, \mathbf{C N}(\mathbf{m M})$ \\
\hline \hline PspE & 9,428 & 64 & 3.4 & 32 \\
& $(85)$ & & 78 & 17 \\
\hline
\end{tabular}

mutant strain revealed apparent co-regulation of $p s p E$ with the preceding genes of the operon [53]. Previous studies also concluded that PspE is regulated independently from the other members of the $p s p$ operon, since a short, $p s p E$ specific transcript was observed that is thought to originate from an independent, $\sigma^{70}$-dependent promoter presumably located within the 75-bp pspD-pspE intergenic region $[22,38]$. The premise that $p s p E$ is not an essential member of the psp regulon is also supported by the observation that among organisms in which the $p s p A B C$ and $p s p F$ genes are conserved, the $p s p D$ and $p s p E$ genes have limited conservation [23].

Likewise, the present work demonstrates that $g \operatorname{lpE}$ is not an essential part of the $g l p$ regulon, since the mutant strains grew normally on minimal medium containing glycerol. Our earlier work revealed that the main promoter for the $g l p E G R$ operon is not subject to specific regulation by GlpR [19], and analysis of completely sequenced bacterial genomes indicates that organization of $g l p E, \operatorname{glp} G$ and $g l p R$ into an operon is not conserved. Therefore, it is possible that the context of $g l p E$ within the $g l p E G R$ operon of E. coli is fortuitous, and has no physiological relevance.

The catalytic properties of native PspE were similar to those of PspE containing an N-terminal polyhistidine tag [9], and to those of previously characterized "accessible" rhodanese of E. coli [55]. In contrast, the properties of PspE were distinct from those of cytoplasmic rhodanese GlpE (Table 4). The main distinction is that PspE has a much higher affinity for thiosulfate compared to GlpE. This property suggests that thiosulfate is in fact a physiological sulfur donor for PspE, which may not be the case for GlpE. Neither enzyme has a high affinity for cyanide, which indicates that these enzymes would not be effective for cyanide detoxification. Indeed, strains deficient in either or both enzymes were not more sensitive to cyanide, and overexpression of either enzyme did not confer resistance to cyanide (reference [9] and our unpublished results).

The various covalently modified forms of PspE detected by mass spectrometry are explained by the known mechanism of rhodanese catalysis, as well as by known reactions of compounds containing sulfur [56]. Rhodaneses catalyze the transfer of sulfur from thiosulfate by using a two step ping-pong mechanism, whereby a covalent enzyme-sulfur intermediate is formed on the active site cysteine of the enzyme during the first step:

$\mathrm{E}-\mathrm{SH}+\mathrm{SSO}_{3}{ }^{2-} \rightarrow \mathrm{E}-\mathrm{S}-\mathrm{S}^{-}+\mathrm{HSO}_{3}^{-}$

During the second step, a thiophilic acceptor such as cyanide displaces the bound sulfur, returning the enzyme to its active form $(\mathrm{E}-\mathrm{SH})$ :
$\mathrm{E}-\mathrm{S}-\mathrm{S}^{-}+\mathrm{CN}^{-} \rightarrow \mathrm{E}-\mathrm{SH}+\mathrm{SCN}^{-}$

Presumably, PspE carrying a second sulfur atom is generated via further reaction of the enzyme-sulfur intermediate with another molecule of sulfur donor:

$\mathrm{E}-\mathrm{S}-\mathrm{S}^{-}+\mathrm{SSO}_{3}{ }^{2-} \rightarrow \mathrm{E}-\mathrm{S}-\mathrm{SS}^{-}+\mathrm{HSO}_{3}$

The sulfonate form of the enzyme appears to be generated by reaction with thiosulfate, since its abundance increased following incubation of PspE2 with thiosulfate (reaction 4). Alternatively, sulfite generated during the rhodanese reaction would be capable of reaction with an oxidized, disulfide form of PspE (reaction 5):

$$
\begin{aligned}
& \mathrm{E}-\mathrm{S}-\mathrm{S}^{-}+\mathrm{SSO}_{3}{ }^{2-} \rightarrow \mathrm{E}-\mathrm{S}-\mathrm{SSO}_{3}{ }^{2-}+\mathrm{HS}^{-} \\
& \mathrm{E}-\mathrm{S}-\mathrm{S}-\mathrm{E}+\mathrm{HSO}_{3}{ }^{-} \rightarrow \mathrm{E}-\mathrm{S}-\mathrm{SO}_{3}{ }^{2-}+\mathrm{E}-\mathrm{SH}
\end{aligned}
$$

The basis for activation of PspE by thiol reagents such as cysteine or dithiothreitol is that these reagents will not only reduce disulfide-linked PspE dimers (reaction 6), but should also react with the sulfonate carried by the active site cysteine or cysteine persulfide (reactions 7 and 8 [56]):

$$
\begin{aligned}
& \text { E-S-SO }{ }_{3}{ }^{-}+\mathrm{R}-\mathrm{SH} \rightarrow \mathrm{E}-\mathrm{S}-\mathrm{S}-\mathrm{R}+\mathrm{HSO}_{3}{ }^{-} \\
& \text {E-S-SSO } \\
& { }^{-}+\mathrm{R}-\mathrm{SH} \rightarrow \mathrm{E}-\mathrm{S}-\mathrm{S}-\mathrm{R}+\mathrm{SSO}_{3}{ }^{2-} \\
& \text { E-S-SSO } \\
& { }^{-}
\end{aligned}
$$

The active form of the enzyme (E-SH or E-SSH) would be generated by intramolecular reduction of the mixed disulfide (in the case of $\mathrm{R}=$ dithiothreitol) or by reaction with a second molecule of cysteine (R-SH, reaction 9):

$\mathrm{E}-\mathrm{S}-\mathrm{S}-\mathrm{R}+\mathrm{R}-\mathrm{SH} \rightarrow \mathrm{E}-\mathrm{SH}+\mathrm{R}-\mathrm{S}-\mathrm{S}-\mathrm{R}$

It should be pointed out that the active form of the enzyme may be the cysteine persulfide form of the enzyme. The persulfide sulfur is apparently quite resistant to removal by cyanide, as demonstrated by the fact that very little sulfur was removed from fraction PspE2 (Fig. 4) even though the persulfide derivative was the most abundant form of PspE found in this fraction (Table 3). It has been observed that the persulfide forms of GlpE and RhdA are stable and may be the active forms of those enzymes [17,57].

The covalent modifications of the active site cysteine of PspE are likely to render the enzyme much less susceptible to oxidation or modification by thiol reagents. The stability of covalently modified PspE may be of physiological relevance, since it is present in the periplasmic space, an oxidizing environment.

The physiological function of PspE remains to be determined. It is possible that PspE participates in trafficking of sulfur needed for formation or repair of sulfur-containing clusters of enzymes within the periplasm, as suggested ear- 
lier [9]. PspE should be suited for such a role, since it is capable of storing multiple sulfur atoms at each active site.

\section{ACKNOWLEDGEMENTS}

This work was supported by a grant from the Thomas F. Jeffress and Kate Miller Jeffress Memorial Trust, and from an ASPIRES award from Virginia Tech. We thank P. Model for providing plasmid pPS-3 and F. Ahmed for strain FA035.

\section{REFERENCES}

[1] Bordo D, Bork P. The rhodanese/Cdc25 phosphatase superfamily: Sequence-structure-function relations. EMBO Rep 2002; 3: 741746.

[2] Cipollone R, Ascenzi P, Visca P. Common themes and variations in the rhodanese superfamily. IUBMB Life 2007; 59: 51-59.

[3] Bordo D, Deriu D, Colnaghi R, et al. The crystal structure of a sulfurtransferase from Azotobacter vinelandii highlights the evolutionary relationship between the rhodanese and phosphatase enzyme families. J Mol Biol 2000; 298: 691-704.

[4] Spallarossa A, Forlani F, Carpen A, et al. The "rhodanese" fold and catalytic mechanism of 3-mercaptopyruvate sulfurtransferases: crystal structure of SseA from Escherichia coli. J Mol Biol 2004; 335: 583-593.

[5] Cipollone R, Bigotti MG, Frangipani E, Ascenzi P, Visca P. Characterization of a rhodanese from the cyanogenic bacterium Pseudomonas aeruginosa. Biochem Biophys Res Commun 2004; 325: 85-90.

[6] Cipollone R, Ascenzi P, Frangipani E, Visca P. Cyanide detoxification by recombinant bacterial rhodanese. Chemosphere 2006; 63: 942-949.

[7] Cipollone R, Frangipani E, Tiburzi F, et al. Involvement of Pseudomonas aeruginosa rhodanese in protection from cyanide toxicity. Appl Environ Microbiol 2007; 73: 390-398.

[8] Ray WK, Potters MB, Mansuri AM, Larson TJ. Characterization of a 12-kilodalton rhodanese encoded by glpE of Escherichia coli and its interaction with thioredoxin. J Bacteriol 2000; 181: 2277-2284.

[9] Adams H, Teertstra W, Koster M, Tommassen J. PspE (phageshock protein E) of Escherichia coli is a rhodanese. FEBS Lett 2002; 518: 173-176.

[10] Palenchar PM, Buck CJ, Cheng H, Larson TJ, Mueller EG. Evidence that ThiI, an enzyme shared between thiamin and 4thiouridine biosynthesis, may be a sulfurtransferase that proceeds through a persulfide intermediate. J Biol Chem 2000; 275: 82838286.

[11] Mueller EG, Palenchar PM, Buck CJ. The role of the cysteine residues of ThiI in the generation of 4-thiouridine in tRNA. J Biol Chem 2001; 276: 33588-33595.

[12] Matthies A, Rajagopalan KV, Mendel RR, Leimkühler S. Evidence for the physiological role of a rhodanese-like protein for the biosynthesis of the molybdenum cofactor in humans. Proc Natl Acad Sci USA 2004; 101: 5946-5951.

[13] Matthies A, Nimtz M, Leimkühler S. Molybdenum cofactor biosynthesis in humans: Identification of a persulfide group in the rhodanese-like domain of MOCS3 by mass spectrometry. Biochemistry 2005; 44: 7912-7920.

[14] Wolfe MD, Ahmed F, Lacourciere GM, et al. Functional diversity of the rhodanese homology domain: the Escherichia coli ybbB gene encodes a selenophosphate-dependent tRNA 2-selenouridine synthase. J Biol Chem 2004; 279: 1801-1809.

[15] Sepulveda-Torres LdC, Huang A, Kim H, Criddle CS. Analysis of regulatory elements and genes required for carbon tetrachloride degradation in Pseudomonas stutzeri strain KC. J Mol Microbiol Biotechnol 2002; 4: 151-161.

[16] Godert AM, Jin M, McLafferty FW, Begley TP. Biosynthesis of the thioquinolobactin siderophore: an interesting variation on sulfur transfer. J Bacteriol 2007; 189: 2941-2944.

[17] Spallarossa A, Donahue JL, Larson TJ, Bolognesi M, Bordo D. Escherichia coli GlpE is a prototype for single-domain sulfurtransferase (rhodanese) homology superfamily. Structure 2001; 9: 11171125 .

[18] Colnaghi R, Cassinelli G, Drummond M, Forlani F, Pagani S. Properties of the Escherichia coli rhodanese-like protein SseA: contribution of the active-site residue Ser240 to sulfur donor recognition. FEBS Lett 2001; 500: 153-156.
[19] Yang B, Larson TJ. Multiple promoters are responsible for transcription of the $g l p E G R$ operon of Escherichia coli K-12. Biochim Biophys Acta 1998; 1396: 114-126.

[20] Lemberg MK, Freeman M. Functional and evolutionary implications of enhanced genomic analysis of rhomboid intramembrane proteases. Genome Res 2007; 17: 1634-1646.

[21] Zeng G, Ye S, Larson TJ. Repressor for the sn-glycerol 3phosphate regulon of Escherichia coli K-12. Primary structure and identification of the DNA-binding domain. J Bacteriol 1996; 178: 7080-7089.

[22] Model P, Jovanovic G, Dworkin J. The Escherichia coli phageshock-protein ( $p s p$ ) operon. Mol Microbiol 1997; 24: 255-261.

[23] Darwin AJ. The phage-shock-protein response. Mol Microbiol 2005; 57: 621-628.

[24] Kobayashi H, Yamamoto M, Aono R. Appearance of a stressresponse protein, phage-shock protein A, in Escherichia coli exposed to hydrophobic organic solvents. Microbiology 1998; 144: 353-359.

[25] Jovanovic G, Lloyd LJ, Stumpf MPH, Mayhew AJ, Buck M. Induction and function of the phage shock protein extracytoplasmic stress response in Escherichia coli. J Biol Chem 2006; 281: 2114721161.

[26] Miller JH. A short course in bacterial genetics. A laboratory manual and handbook for Escherichia coli and related bacteria. Cold Spring Harbor Laboratory Press: Plainview, N.Y., 1992.

[27] Xi J, Ge Y, Kinsland C, McLafferty FW, Begley TP. Biosynthesis of the thiazole moiety of thiamin in Escherichia coli: Identification of an acyldisulfide-linked protein-protein conjugate that is functionally analogous to the ubiquitin/E1 complex. Proc Natl Acad Sci USA 2001; 98: 8513-8518.

[28] Johnson ME, Rajagopalan KV. Involvement of $\operatorname{chlA}, E, M$, and $N$ loci in Escherichia coli molybdopterin biosynthesis. J Bacteriol 1987; 169: 117-125.

[29] Blattner FR, Plunkett G, Bloch CA, et al. The complete genome sequence of Escherichia coli K-12. Science 1997; 277: 1453-1462.

[30] Studier FW, Moffatt BA. Use of bacteriophage T7 RNA polymerase to direct selective high-level expression of cloned genes. $\mathbf{J}$ Mol Biol 1986; 189: 113-130.

[31] Lutz R, Bujard H. Independent and tight regulation of transcriptional units in Escherichia coli via the LacR/O, the TetR/O and AraC/I $I_{1}-I_{2}$ regulatory elements. Nucleic Acids Res 1997; 25: 12031210.

[32] Nichols BP, Shafiq O, Meiners V. Sequence analysis of $\operatorname{Tn} 10$ insertion sites in a collection of Escherichia coli strains used for genetic mapping and strain construction. J Bacteriol 1998; 180: 64086411.

[33] Donahue JL, Bownas JL, Niehaus WG Jr, Larson TJ. Purification and characterization of $g l p X$-encoded fructose 1,6-bisphosphatase, a new enzyme of the glycerol 3-phosphate regulon of Escherichia coli. J Bacteriol 2000; 182: 5624-5627.

[34] Silhavy TJ, Berman ML, Enquist LW. Experiments with Gene Fusions. Cold Spring Harbor Laboratory: Cold Spring Harbor, N.Y., 1984.

[35] Datsenko KA, Wanner BL. One-step inactivation of chromosomal genes in Escherichia coli K-12 using PCR products. Proc Natl Acad Sci USA 2000; 97: 6640-6645.

[36] Cherepanov PP, Wackernagel W. Gene disruption in Escherichia coli: $\mathrm{Tc}^{\mathrm{R}}$ and $\mathrm{Km}^{\mathrm{R}}$ cassettes with the option of Flp-catalyzed excision of the antibiotic-resistance determinant. Gene 1995; 158: 9-14.

[37] Wolfe MD, Ahmed F, Lacourciere GM, et al. Functional diversity of the rhodanese homology domain: Escherichia coli $\mathrm{YbbB}$ is a selenophosphate-dependent tRNA 2-selenouridine synthase. J Biol Chem 2004; 279: 1801-1809.

[38] Brissette JL, Weiner L, Ripmaster TL, Model P. Characterization and sequence of the Escherichia coli stress-induced psp operon. J Mol Biol 1991; 220: 35-48.

[39] Laemmli U. Cleavage of structural proteins during the assembly of the head of bacteriophage T4. Nature 1970; 227: 680-685.

[40] Westley J. Thiosulfate: cyanide sulfurtransferase (rhodanese). Methods Enzymol 1981; 77: 285-291.

[41] Jarabak R. 3-Mercaptopyruvate sulfurtransferase. Methods Enzymol 1981; 77: 291-297.

[42] Wood JL. Sulfane sulfur. Methods Enzymol 1987; 143: 25-29.

[43] Roy AB, Trudinger PA. Rhodanese and 3-mercaptopyruvate sulfurtransferase. In: The Biochemistry of Inorganic Compounds of Sulfur. Cambridge, Cambridge University Press. 1970; 190-206. 
[44] Stafford SJ, Humphreys DP, Lund PA. Mutations in $d s b A$ and $d s b B$, but not $d s b C$, lead to an enhanced sensitivity of Escherichia coli to $\mathrm{Hg}^{2+}$ and $\mathrm{Cd}^{2+}$. FEMS Microbiol Lett 1999; 174: 179-184.

[45] Rensing C, Mitra B, Rosen BP. Insertional inactivation of $d s b A$ produces sensitivity to cadmium and zinc in Escherichia coli. J Bacteriol 1997; 179: 2769-2771.

[46] Glasner JD, Rusch M, Liss P, et al. ASAP: a resource for annotating, curating, comparing, and disseminating genomic data. Nucleic Acids Res 2006; 34: D41-D45.

[47] Glasner JD, Liss P, Plunkett G III, et al. ASAP, a systematic annotation package for community analysis of genomes. Nucleic Acids Res 2003; 31: 147-151.

[48] Wasinger VC, Humphery-Smith I. Small genes/gene-products in Escherichia coli K-12. FEMS Microbiol Lett 1998; 169: 375-382.

[49] Pensa B, Costa M, Pecci L, Cannella C, Cavallini D. Selective reactivity of rhodanese sulfhydryl groups with 5,5'-dithio-bis(2nitrobenzoic acid). Biochim Biophys Acta 1977; 484: 368-374.

[50] Bradford MM. A rapid and sensitive method for the quantitation of microgram quantities of protein utilizing the principle of proteindye binding. Anal Biochem 1976; 72: 248-254.

[51] Islam TA, Miller-Martini DM, Horowitz PM. Mutation of cysteine 254 facilitates the conformational changes accompanying the inter- conversion of persulfide-substituted and persulfide-free rhodanese. J Biol Chem 1994; 269: 7908-7913.

[52] Westley J, Heyse D. Mechanisms of sulfur transfer catalysis. Sulfhydryl-catalyzed transfer of thiosulfonate sulfur. J Biol Chem 1971; 246: 1468-1474.

[53] Lloyd LJ, Jones SE, Jovanovic G, et al. Identification of a new member of the phage shock protein response in Escherichia coli, the phage shock protein G (PspG). J Biol Chem 2004; 279: 5570755714.

[54] Waterman DG, Ortiz-Lombardia M, Fogg MJ, Koonin EV, Antson AA. Crystal structure of Bacillus anthracis ThiI, a tRNAmodifying enzyme containing the predicted RNA-binding THUMP domain. J Mol Biol 2006; 356: 97-110.

[55] Alexander K, Volini M. Properties of an Escherichia coli rhodanese. J Biol Chem 1987; 262: 6595-6604.

[56] Roy AB, Trudinger PA. The chemistry of some sulfur compounds. In: The Biochemistry of Inorganic Compounds of Sulfur. Cambridge, Cambridge University Press. 1970; 7-42.

[57] Bordo D, Forlani F, Spallarossa A, et al. A persulfurated cysteine promotes active site reactivity in Azotobacter vinelandii rhodanese. Biol Chem 2001; 382: 1245-1252. 